\title{
Frequency-sweep generation by resonant self-injection locking
}

\author{
Joerg Troger, Luc Thévenaz, and Philippe Robert \\ Laboratory of Metrology, Swiss Federal Institute of Technology, CH-1015 Lausanne, Switzerland
}

Received June 25, 1999

\begin{abstract}
We report a novel application of self-injection locking. A diode laser is injection locked to its own frequencyshifted emission. By resonant phase modulation of the fed-back light, the laser's emission frequency is shown to swing periodically through the locking range. The laser operates as a sweep generator driven by resonant self-injection locking. (c) 1999 Optical Society of America

OCIS codes: $\quad 140.3520,140.3490,060.2430,060.2310,060.2630,060.5060$.
\end{abstract}

Injection locking ${ }^{1-6}$ and passive feedback ${ }^{7-9}$ are closely related topics. In the case of injection locking, a signal from an external source (i.e., the master laser) is injected into the cavity of another laser (i.e., the slave laser). In contrast, in passive feedback setups, the time-delayed (coherent or incoherent) signal from a laser is fed back into its own cavity. In short, the slave laser is its own master laser (and vice versa). The physical phenomena observed in both configurations are similar and range from linewidth narrowing and undamped relaxation oscillations to chaotic behavior.

In this Letter we discuss a special application of injection locking that, in essence, is a passive feedback experiment. The slave-laser emission is delayed in time, frequency shifted in a phase modulator, and fed back into the laser cavity. The laser then locks on its own frequency-shifted signal. From the injectionlocking point of view, this behavior can be viewed as a kind of self-injection locking. Under resonant phase modulation, the slave laser's instantaneous frequency can be seen to swing back and forth through the locking range. Feedback effects in semiconductor lasers have been investigated in many works. ${ }^{7-9}$ Yet, to our knowledge, resonant frequency-shifted feedback has not been treated in the literature.

Figure 1 shows the experimental setup of the sweep generator. The slave laser is a $1.55-\mu \mathrm{m}$ multiplequantum-well graded-index separate confinement heterostructure distributed-feedback device with a threshold current of $25 \mathrm{~mA}$. The laser is biased at $I=100 \mathrm{~mA}$ and emits $P_{\text {out }}$ fr $=8.8 \mathrm{~mW}$ per facet. Its coherence length is approximately $100 \mathrm{~m}$. Light from the laser passes through the circulator through the delay line and is frequency shifted in the modulator. A part of the light is then coupled back into the laser. As a consequence of the long delay line, the fed-back light is uncorrelated with the emitted light. The slave-laser spectrum is analyzed by use of a Fabry-Perot scanning interferometer. In FC 4, the slave-laser radiation is mixed with the emission from a reference laser. A fast oscilloscope records the temporal evolution of the resulting beat-note intensity. The reference laser is tuned to emit at the frequency of the free-running (i.e., without feedback) slave laser. The factor $K$ is the light-coupling efficiency between the slave laser and the optical fiber. By reciprocity, $K$ also describes the coupling of light between the fiber and the slave-laser cavity. It is given by $K=P_{(\mathrm{A})} /\left(P_{\text {out }}{ }^{\text {fr }} \eta\right)$, where $P_{(\mathrm{A})}$ is the optical power measured at point $(\mathrm{A})$ when the fiber connectors are temporarily opened. $\eta=0.958$ is the fraction of uncoupled power in $\mathrm{FC} 1$, in which excess loss is negligible. In our configuration, typical values of $K$ are near 0.3 , with good alignment of the optical elements. PC 2 is required to optimize the operation of the phase modulator. After PC 1 and PC 2 are tuned, the feedback level is fixed with PC 3 and monitored on the optical powermeter [point (D)].

The polarizations of the slave-laser intracavity and injected fields have to be identical for maximum interaction and avoidance of polarization switching between TE and TM modes. Polarization matching is achieved as follows: The output radiation from the slave laser is polarized linearly. Branch (B) of FC 2 monitors the part of the power that is transmitted through the fiber polarizer. We maximize the transmission by tuning $\mathrm{PC}$ 1. In this condition the slave-laser light is also linearly polarized at point $(\mathrm{C})$. Then, in the absence of any nonreciprocal effects, the polarizations of the injected light from the master-laser and the slave-laser intracavity fields are identical and linear.

Figure 2 illustrates the temporal and spectral behaviors of the self-injection-locked slave laser. There is no

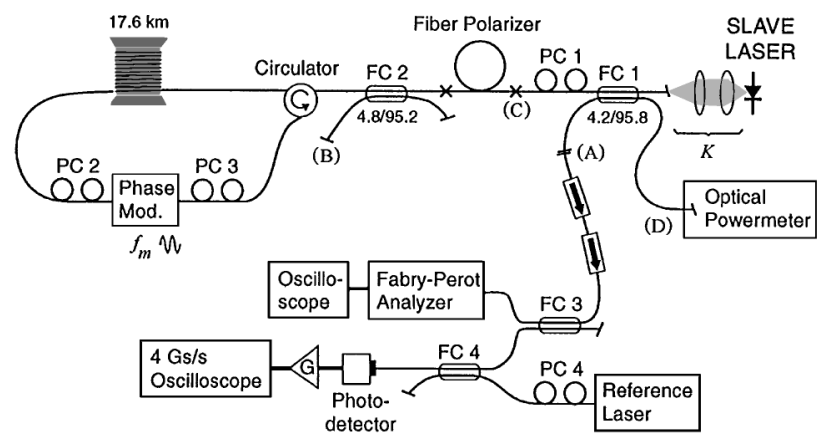

Fig. 1. Setup of the frequency-sweep generator experiment: The slave-laser radiation travels through $17.6 \mathrm{~km}$ of single-mode fiber and is phase modulated before a part of it is fed back into the slave laser. FC 1-FC 4, fiber couplers; PC 1-PC 4, polarization controllers. Mod., modulator; G, amplifier. Gs/s, gigasamples per second. The two isolators separate the sweep generator from the measuring instruments.

(C) 1999 Optical Society of America 
(a)
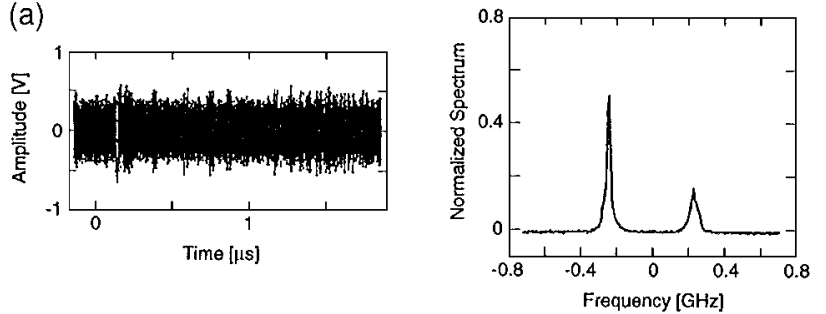

(b)

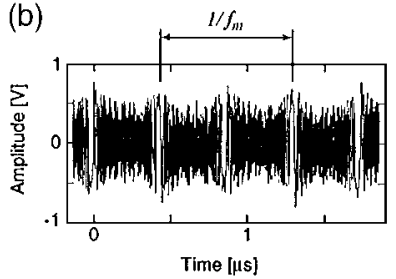

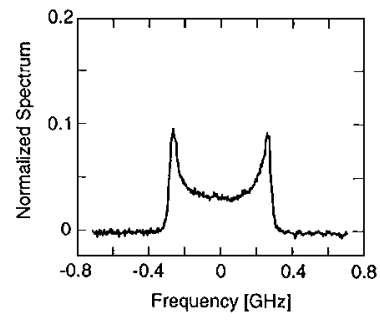

Fig. 2. Time evolution of beat-note intensity (ac component) and related power spectrum of the self-injectionlocked slave laser (a) without phase modulation and (b) with sinusoidal resonant phase modulation $\left(V_{m} / V_{\pi} \approx\right.$ $\left.0.27, f_{m}=1.15 \mathrm{MHz}\right)$. The feedback level is $P_{\text {inj }} / P_{\text {out }}$ fr $\approx$ $9.0 \times 10^{-5}$. The frequency 0 refers to the emission frequency of the free-running slave and the reference laser. The spectra are normalized with respect to the power of the free-running slave laser.

phase modulation in the case shown in Fig. 2(a), but there is resonant phase modulation in the case shown in Fig. 2 (b). The light's round-trip time in the $L_{\text {ext }} \approx$ $17.6 \mathrm{~km}$ fiber loop amounts to $\tau_{\mathrm{ext}} \approx 87 \mu \mathrm{s}$, which gives a fundamental resonant frequency of $f_{0}=1 / \tau_{\text {ext }} \approx$ $11.5 \mathrm{kHz}$. The feedback level is set at $P_{\text {inj }} / P_{\text {out }}$ fr $\approx$ $9.0 \times 10^{-5}$. Note that $P_{\text {inj }}$ is defined as the fed-back power at the slave-laser interface that matches the mode of the laser waveguide and $P_{\text {out }}{ }^{\text {fr }}$ is the output power per facet of the free-running slave laser. Dynamic instability ${ }^{2-6}$ occurs at $P_{\text {inj }} /\left.P_{\text {out }}{ }_{\text {fr }}\right|_{\text {inst }} \approx 2 \%$, that is, well above the actual feedback level.

Consider first the experiment without phase modulation: Fig. 2(a) shows that the beat-note intensity between the slave- and the reference-laser radiations varies irregularly with time. The power spectrum contains two asymmetric peaks and extends approximately $\pm 0.3 \mathrm{GHz}$, which is the locking range for the given feedback.

Next we let spontaneous emission be neglected initially and refer to Fig. 3. The figure displays the calculated variations in the injection-locked slave-laser carrier number and output power as a function of the frequency offset at $P_{\text {inj }} / P_{\text {out }}$ fr $\approx 9.0 \times 10^{-5}$. Before the feedback is turned on, the slave laser emits at its free-running frequency (that is, at $\Delta \nu=0$, as shown in Fig. 3). As the feedback is switched on, some light returns from the first round trip in the fiber loop, is injected back into the laser cavity, and gives rise to increased laser output power [see Fig. 3(b)]. This increased output power means a decreased carrier number [see Fig. 3(a)] and, therefore, an increased refractive index of the waveguide. Hence, the laser's emission frequency is lowered as a consequence of the fed-back light. After each round trip, the slave laser injection locks itself nearly instantaneously to the light that it emitted one round-trip time earlier. The transients that are related to the injection-locking mechanism typically last less than $10 \mathrm{~ns}^{4} \quad$ Thus, the laser's emission frequency is subsequently lowered until the laser reaches the edge of the locking range. When the laser arrives at the lower edge of the locking range, the self-injection-locking mechanism ceases, and the slave laser keeps lasing at frequencies nearby. This continued lasing explains the spectral peak at $-0.25 \mathrm{GHz}$ in Fig. 2(a) near the lower edge of the locking range.

Now, consider the effect of spontaneous emission. Assume that the laser emits near the lower edge of the locking range. Rare but large phase and power fluctuations in response to spontaneous-emission events ${ }^{10}$ may drive the laser's instantaneous frequency to a point at which the laser's output power decreases compared with that of the free-running device [Fig. 3(b) shows that this is the case when $\Delta \nu>0.1 \mathrm{GHz}$. If this happens, the increased carrier population leads to a reduced refractive index and hence to an increased laser frequency. Then, as a consequence of alternating self-injection locking and frequency shifting, the laser's emission frequency grows continuously and finally finds itself at the upper edge of the locking range. In Fig. 2(a), the spectral peak near $+0.25 \mathrm{GHz}$ is formed. In this respect, the spectrum in Fig. 2(a) shows that the peak at $-0.25 \mathrm{GHz}$ is significantly stronger than the peak at $+0.25 \mathrm{GHz}$. Hence, the slave laser spends on average more time emitting near the lower edge of the locking range than near the upper edge. This behavior can again be explained with the help of Fig. 3. Owing to the asymmetries in the curves (in particular, the zero crossings do not occur at zero detuning), it is more probable that spontaneous emission drives the slave-laser frequency to the lower edge of the locking range than to the upper edge. This is the reason for the dominant spectral peak near the lower edge of the locking range.

In the measurement shown in Fig. 2(b), the phase modulator is operated sinusoidally at the resonant frequency $f_{m}=100 f_{0} \approx 1.15 \mathrm{MHz}$ and at a normalized voltage $V_{m} / V_{\pi} \approx 0.27 . \quad V_{\pi}$ is the peak-to-peak voltage that is required for a $\pi$ phase shift in the modulator. The temporal evolution of the beat-note intensity has a periodic pattern. The periodic transitions of the slave (a)

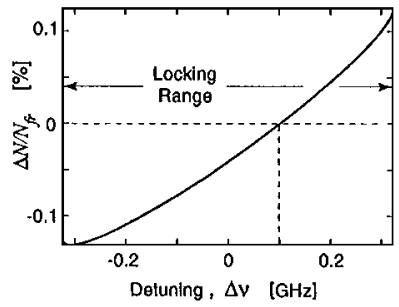

(b)

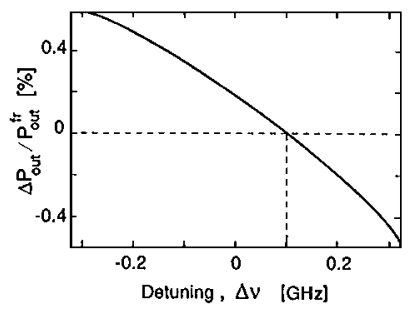

Fig. 3. Calculated variations over the locking range owing to feedback $\left(P_{\text {inj }} / P_{\text {out }}{ }^{\mathrm{fr}} \approx 9.0 \times 10^{-5}\right)$ of (a) the active region carrier number and (b) the output power. $\Delta \nu$ is the frequency difference between the fed-back signal and the output radiation of the free-running slave laser. 


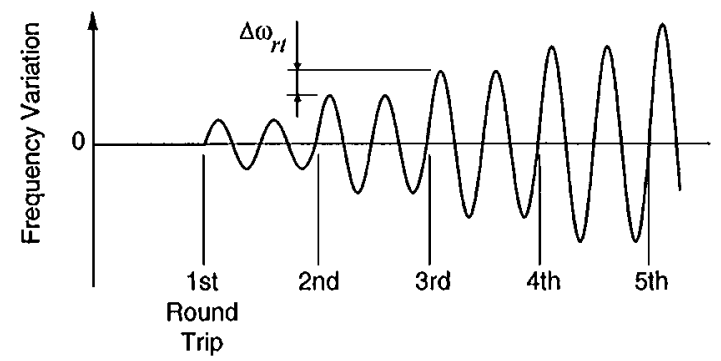

Fig. 4. Instantaneous frequency variation of the fed-back light at the laser input after $1,2,3, \ldots$ round trips in the fiber loop. Owing to resonant phase modulation, the frequency variations add up constructively at each round trip, $f_{m}=2 f_{0}$ in the illustrated case. 0 indicates the emission frequency of the free-running laser. The refractive-index-induced frequency variations are omitted.

laser's instantaneous frequency across the reference frequency show up in the figure as white stripes. The power spectrum takes the characteristic shape of a frequency-modulated laser with two symmetric peaks at approximately $\pm 0.25 \mathrm{GHz}$.

At each round trip, the phase modulator increases the instantaneous frequency sweep of the traveling light wave proportionally to the modulation amplitude and frequency. Under sinusoidal phase modulation, the time-dependent phase variation in the modulator can be written as $\Delta \phi_{m}(t)=\pi\left(V_{m} / V_{\pi}\right) \sin \left(2 \pi f_{m} t\right)$. The resulting instantaneous angular frequency variation is given by $\Delta \omega_{m}=2 \pi^{2}\left(V_{m} / V_{\pi}\right) f_{m} \cos \left(2 \pi f_{m} t\right)$. Hence, under resonant modulation, the amplitude of the instantaneous frequency sweep increases by $\Delta \omega_{\mathrm{rt}}=$ $2 \pi^{2}\left(V_{m} / V_{\pi}\right) f_{m}$ per round trip. In Fig. $2(\mathrm{~b}), V_{m} / V_{\pi} \approx$ 0.27 and $f_{m}=1.15 \mathrm{MHz}$, yielding an increment of 6.1 MHz. When the modulator is operated at the fundamental resonant frequency, $f_{m}=f_{0} \approx 11.5 \mathrm{kHz}$, instead, the increment per round trip is $61 \mathrm{kHz}$, which is not sufficient for the sweep generator to work properly, as is explained below.

Resonant phase modulation is pictured in Fig. 4, in which the above-mentioned frequency shifts owing to the refractive-index change are not shown. The slave laser continuously locks on its own frequencymodulated signal until the built-up frequency sweep exceeds the locking range. At this point, the selfinjection locking cannot be maintained any further. The laser's instantaneous frequency then keeps swinging periodically back and forth through the locking range, at a rate determined by the modulation frequency $f_{m}$. Resonant phase modulation is necessary for the frequency deviations per round trip to add up in phase. If the modulation is not resonant, the individual frequency shifts per round trip compensate for one another, and the slave laser does not swing periodically through the full locking range.

Our measurements showed that the lower limit for the round-trip frequency increment was of the order of $1 \mathrm{MHz}$. In fact, for the sweep generator to work properly, the frequency increment that is due to phase modulation must outweigh the frequency shift that is induced by the refractive-index change. Moreover, we assume that the upper limit for the phase-modulation frequency is given by the injection-locking response time. It should, therefore, lie in the $100-\mathrm{MHz}$ range.

Experimentally, the swept frequency range was shown to increase with the feedback level. However, the maximum feedback that was achieved was only approximately $P_{\text {inj }} / P_{\text {out }}^{\text {fr }} \approx 1 \%$, owing to the high coupling loss between the slave laser and the fiber and the significant losses in the fiber loop. Sweep ranges of as much as $2 \mathrm{GHz}$ were found. Theoretically, with the slave laser biased at $100 \mathrm{~mA}$, it should be possible to extend the swept frequency range to $10 \mathrm{GHz}$, where the injection-locking instability edge is reached $\left(P_{\text {inj }} /\left.P_{\text {out }}{ }^{f r}\right|_{\text {inst }} \approx 2 \%\right)$. Several measurements with shorter delay lines were made. In particular, the fiber loop length was reduced to $47 \mathrm{~m}$ by suppression of the $17.6-\mathrm{km}$ fiber. The sweep generator also worked in this case of coherent optical feedback.

In conclusion, we have presented an optical frequency-sweep generator that is based on the principle of frequency-shifted, resonant self-injection locking. The swept frequency range is determined by the feedback level and typically amounts to $1 \mathrm{GHz}$. Sweep frequencies of several tens of megahertz are achieved.

\section{J. Troger's e-mail address is joerg.troger@epfl.ch.}

\section{References}

1. S. Kobayashi and T. Kimura, IEEE J. Quantum Electron. QE-17, 681 (1981).

2. R. Lang, IEEE J. Quantum Electron. QE-18, 976 (1982).

3. C. H. Henry, N. A. Olsson, and N. K. Dutta, IEEE J. Quantum Electron. QE-21, 1152 (1985).

4. P. Spano, M. Tamburrini, and S. Piazzolla, J. Lightwave Technol. 7, 726 (1989).

5. R. Hui, A. D’Ottavi, A. Mecozzi, and P. Spano, IEEE J. Quantum Electron. 27, 1688 (1991).

6. J. Troger, P.-A. Nicati, L. Thévenaz, and Ph. A. Robert, IEEE J. Quantum Electron. 35, 32 (1999).

7. R. Lang and K. Kobayashi, IEEE J. Quantum Electron. QE-16, 347 (1980).

8. D. Lenstra, B. H. Verbeek, and A. J. den Boef, IEEE J. Quantum Electron. QE-21, 674 (1985).

9. C. H. Henry and R. F. Kazarinov, IEEE J. Quantum Electron. QE-22, 294 (1986).

10. C. H. Henry, J. Lightwave Technol. 4, 298 (1986). 